\title{
Epidemiological Study of Periodontal Disease and White Blood Cell Count among Employees in a Company
}

\author{
Yukie Yoshida ${ }^{1}$, Masahide Imaki ${ }^{1}$, Ken Nishida' and Seiki Tanada ${ }^{2}$ \\ 'Osaka Prefectural College of Health Science and \\ ${ }^{2}$ Faculty of Pharmaceutical Sciences, Kinki University
}

\begin{abstract}
Epidemiological Study of Periodontal Disease and White Blood Cell Count among Employees in a Company: Yukie YosHIDA, et al. Osaka Prefectural College of Health Science-The purpose of this study was to investigate the relationship between the white blood cell (WBC) count and periodontal disease as classified by the Community Periodontal Index of Treatment Needs (CPITN) in 1,241 male Japanese factory workers by determining their WBC count and the extent of their periodontal disease. The results obtained were as follows: in both smoker and non-smoker, the results of a two-way ANOVA indicated that the CPITN score 3 and 4 groups had a significantly higher WBC count than the CPITN score 0,1 and 2 groups. The results of multiple regression analysis indicated that the WBC counts were significantly associated with age, smoking and the CPITN score. It appeared that there was a significant relationship between the WBC count and the prevalence of periodontal disease among employees in a company.

(J Occup Health 1997; 39: 92-94)
\end{abstract}

Key words: Periodontal disease, Coronary heart disease, White blood cell count, CPITN

In Japan, the mortality rate from heart disease has been steadily increasing since 1950 , and in 1985 it exceeded that of cerebrovascular diseases. For the effective prevention of heart disease, it is essential to evaluate the effects of various lifestyle factors for heart disease ${ }^{1}$.

The development of coronary heart disease is attributable to several risk factors such as a high serum total cholesterol concentration, low serum high-density lipoprotein cholesterol concentration, smoking, hypertension and diabetes $^{2}$. These risk factors, however, seem to explain only $50-75 \%$ of the variation in the incidence of coronary heart disease cases in most industrialized societies ${ }^{3)}$. Some important risk factors therefore do not seem to have yet

Received May 1, 1996; Accepted Aug 30, 1996

Correspondence to: $Y$. Yoshida, Osaka Prefectural College of Health Science, Habikino 3-7-30, Habikino 583, Japan been identified.

The white blood cell (WBC) count has been demonstrated in several epidemiologic studies to be a strong independent predictor of future coronary heart disease ${ }^{4)}$. Although it is not yet possible to be certain that the high WBC count is a cause rather than a consequence of ischemic heart disease, recent pathophysiologic studies suggest that the $\mathrm{WBC}$, in particular the neutrophil, is instrumental in the pathogenesis of myocardial ischemia ${ }^{5}$.

Several studies have shown that dental disease was worse in survivors of myocardial infarction than in controls ${ }^{6,7)}$. This association was not explained by the aforementioned risk factors. One possible explanation for this association is that the presence of chronic oral bacterial infection, in the form of gingivitis or periodontitis, could increase plasma fibrinogen and the WBC count, which are consistent, strong predictors of coronary heart disease ${ }^{8)}$. Gingivitis and periodontitis are two main forms of periodontal disease and may be important determinants of variations in fibrinogen levels and the WBC count within the general population.

In the present study, therefore, we examined approximately 1,200 male Japanese factory workers, and investigated the relationship between the oral health indicated by the Community Periodontal Index of Treatment Needs (CPITN) ${ }^{9)}$ and the WBC count.

\section{Population and Methods}

The 1,241 subjects were male factory workers employed at a chemical factory in Osaka, Japan. Table 1 shows the number of subjects by age and cigarette smoking status. None of the subjects was exposed to any toxic substance in the workplace, and those in whom subsequent physical examination and laboratory blood tests (liver function, blood cell count, total cholesterol level, fasting blood sugar) and urine tests (protein, sugar, occult blood) results revealed the presence of any disease, including symptoms of the common cold or the consumption of medicine, were excluded. Subjects with a history of cardiovascular disease, diabetes, hepatitis or any other disease likely to increase the WBC 
count were excluded.

\section{Measurement of oral health and WBC count}

The incidence of periodontal disease as classified by the Community Periodontal Index of Treatment Needs (CPITN) was investigated ${ }^{(1)}$. Periodontal examinations were carried out by a team of trained examiners. All subjects gave their consent to participate after hearing the purpose and details of study.

Blood specimens were collected from a cubital vein in

Table 1. Number of subjects by age and cigarette smoking

\begin{tabular}{crrr}
\hline Age (years) & Smoker & Non-smoker & Total \\
\hline $20-29$ & 217 & 127 & 344 \\
$30-39$ & 191 & 99 & 290 \\
$40-49$ & 225 & 193 & 418 \\
$50-59$ & 89 & 76 & 165 \\
$60-69$ & 11 & 13 & 24 \\
\hline Total & 733 & 508 & 1,241 \\
\hline
\end{tabular}

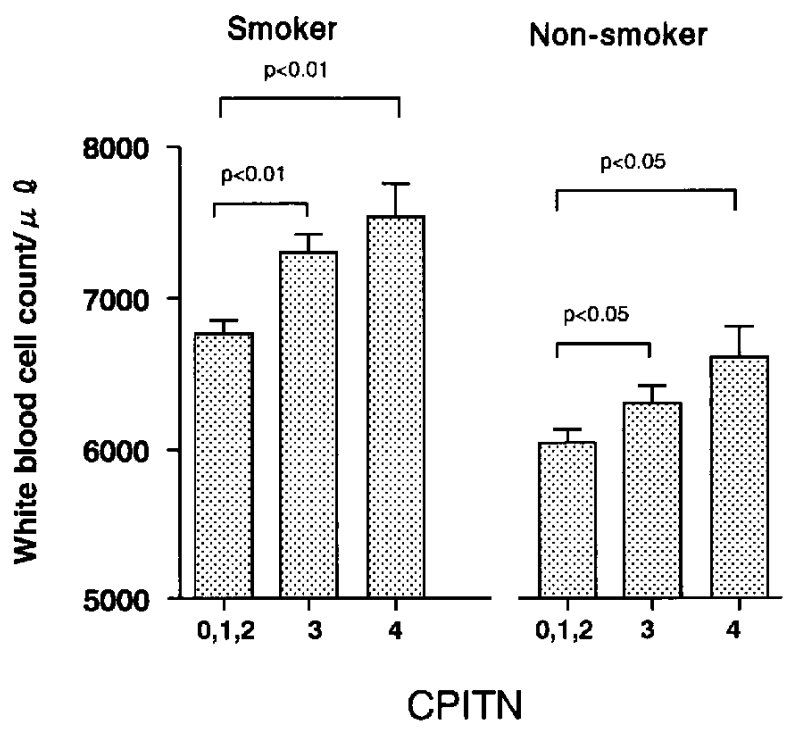

Fig. 1. Mean white blood cell count by CPITN score. the morning before breakfast. The WBC count (Coulter counter) was determined in blood anticoagulated with dipotassium EDTA.

\section{Statistical analysis}

Differences in the WBC count were analyzed by a 2-way analysis of variance (2-ANOVA) in each CPITN group with the effects of age controlled.

A multiple regression analysis was performed in which the dependent variable was the WBC count and the independent variables were age, the CPITN score and cigarette smoking. Significance was defined as $p<0.05$.

\section{Results}

The CPITN score and mean WBC count are shown in Fig. 1. In both smoker and nonsmoker, the results of the 2ANOVA indicated that the CPITN score 3 and 4 groups had a significantly higher WBC count than did the CPITN score 0,1 and 2 groups.

Table 2 shows the results of multiple regression analysis of the WBC count and age, smoking and the CPITN score. There were significantly correlations between the WBC count and age, smoking and the CPITN score. The standardized regression coefficient for smoking was higher than other variable.

\section{Discussion}

The association between dental infections and coronary heart disease is difficult to study. Both diseases are related to the lifestyle, and they share several pathogenic factors such as low socio-economic status ${ }^{10)}$, smoking ${ }^{11)}$ and diabetes ${ }^{12}$. The results of the present study indicated that individuals with gingival or periodontal infection may have a significant increase in their blood white cell count, which is presumably related to the chronic inflammatory nature of these infections ${ }^{8\}}$. Such an increase carries a high relative risk of coronary heart disease, possibly because there are several plausible mechanisms by which the WBC may promote atherosclerosis, thrombosis and myocardial ischemia ${ }^{5}$.

Friedman et al. ${ }^{13)}$ reported a case-control study in which they found that the WBC count was predictive of subsequent myocardial infarction. Kostis et al. ${ }^{147}$ examined the

Table 2. Multiple regression analysis of white blood cell (WBC) count and age, smoking and CPITN

\begin{tabular}{ccccc}
\hline Variables & $\begin{array}{c}\text { Regression } \\
\text { coefficient }\end{array}$ & $\begin{array}{c}\text { Standardized } \\
\text { regression } \\
\text { coefficient }\end{array}$ & t-values & p-values \\
\hline Age & 0.197 & 0.128 & 4.372 & 0.001 \\
Smoking & 4.664 & 0.250 & 9.141 & 0.001 \\
CPITN & 1.156 & 0.063 & 2.147 & 0.032 \\
Constant & 46.096 & 46.096 & 21.203 & \\
\hline
\end{tabular}


relationship between coronary risk factors including the WBC count and angiographic evidence of coronary artery disease in a retrospective study of 573 patients who had undergone cardiac catheterization.

Although leukocytes are traditionally thought of as a defender against infections, they also play an important role in microvascular perfusion. Emst et al. ${ }^{15)}$ have outlined three mechanisms by which leukocytes may contribute to microvascular injury and promote atherosclerosis. These include (1) pressure-dependent plugging of microvessels, (2) rheologic properties such as altered deformability and the formation of aggregates when provoked by a variety of stimuli, and (3) release of activated substances including oxygen-free radicals, proteolytic enzymes, and arachidonic acid metabolites.

In the present study, we observed that although the WBC count was associated with smoking and CPITN, the multiple regression analysis suggested that periodontal disease was an important and independent determinant of the WBC count. Data from the Second Glasgow MONICA study ${ }^{16)}$ also suggested that inter-group differences in age and smoking were insufficient to explain differences in the WBC count.

Our present results suggested that there was a significant association between oral health (expressed as the CPITN score) and the WBC count. Further studies are needed to elucidate the factors and mechanisms by which oral health and coronary heart disease arise and may affect each other.

\section{References}

1) Barnard RJ. Effects of life-style modification on serum lipids. Arch Intern Med 1991; 151: 1389-1394.

2) Carlson L, Böttiger L. Risk factors for ischaemic heart disease in men and women. Acta Med Scand 1985; 218 : 207-211.

3) Spodick DH. Inflammation and the onset of myocardial infarction. Ann Intern Med 1985; 102: 699-702.

4) Hansen LK, Grimm RH, Neaton JD. The relationship of white blood cell count to other cardiovascular risk factors. Intern J Epidemiol 1990; 19: 881-888.

5) Ensrud K, Grimm RH. The white blood cell count and risk for coronary heart disease. Am Heart J 1992; 124: 207-213.

6) Mattila KJ. Dental infections as a risk factor for acute myocardial infarction. Eur Heart J 1993; 14 (Supplement): 51-53.

7) Paunio K, Impivaara O, Tiekso J, Maki J. Missing teeth and ischemic heart disease in men aged $45-64$ years. Eur Heart J 1993; 14 (Supplement): 54-56.

8) Lowe G. Blood rheology in general medicine and surgery. Bailliere's Clin Haematol 1987; 1: 827-861.

9) Cutress TW, Ainamo J, Sardo-Infirri J. The community periodontal index of treatment needs (CPITN) procedure for population groups and individuals. Int Dent J 1987; 37: 222-233.

10) Hausen H, Milen A, Heinonen OP, Paunio I. Caries in primary dentition and social class in high and low fluoride areas. Comm Dent Oral Epidemiol 1982; 10: 33-36.

11) Rivera-Hidalgo F. Smoking and periodontal disease: A review of the literature. J Periodontol 1986; 57: 617-624.

12) Kannel WB. Lipids, diabetes, and coronary heart disease: Insights from the Framingham study. Am Heart J 1985; 110: $1100-1107$.

13) Friedman GD, Klatsky AL, Siegelaub AB. The leukocytes count as a predictor of myocardial infarction. $\mathrm{N}$ Engl $\mathrm{J}$ Med 1974; 290: 1275-1278.

14) Kostis JB, Turkevich D, Sharp J. Association between leukocyte count and the presence and extent of coronary atherosclerosis as determined by coronary arteriography. Am J Cardiol 1984; 53: 997-999.

15) Ernst E, Hammerschmidt DE, Bagge U, Matrai A, Dormandy JA. Leukocytes and the risk of ischemic diseases. JAMA 1987; 257: 2318-2324.

16) Lowe GDO, Lee AJ, Rumley A, Smith WC, Tustall-Pedoe H. Epidemiology of hematocrit, white blood cell, red cell aggregation and fibrinogen: the Glasgow MONICA study. Clin Hemorheol 1992; 12: 535-544. 